\title{
Quantum Processing Photonic States in Optical Lattices
}

\author{
Christine A. Muschik, Inés de Vega, Diego Porras, and J. Ignacio Cirac \\ Max-Planck-Institut für Quantenoptik, Hans-Kopfermann-Strasse, D-85748 Garching, Germany
}

(Received 9 November 2006; published 13 February 2008)

\begin{abstract}
The mapping of photonic states to collective excitations of atomic ensembles is a powerful tool which finds a useful application in the realization of quantum memories and quantum repeaters. In this work we show that cold atoms in optical lattices can be used to perform an entangling unitary operation on the transferred atomic excitations. After the release of the quantum atomic state, our protocol results in a deterministic two qubit gate for photons. The proposed scheme is feasible with current experimental techniques and robust against the dominant sources of noise.
\end{abstract}

DOI: 10.1103/PhysRevLett.100.063601

PACS numbers: 03.67.Lx, 03.67.Ac, 03.67.Bg, 37.10.Jk

Photonic channels are ideally suited for the transmission of quantum states, since current technology is able to distribute photons between remote locations by means of optical fibers. For this reason, they play a key role in practical applications of quantum information such as quantum cryptography. The storage and manipulation of photons is, however, more problematic. Storage of photonic quantum states can be efficiently implemented by interfacing a photonic channel with an atomic system. This idea can be used to realize quantum repeaters [1], and thus, to overcome the problem of losses in the photonic channel by applying entanglement purification at intermediate locations. Manipulation of photonic states requires the ability to perform entangling operations. One possibility is to make use of materials which exhibit optical nonlinearites, but so far, available nonlinearities are too weak to provide us with short gate times. A completely different approach which only requires linear optical operations and measurements was proposed in [2]. However, this scheme is not very efficient in practice.

In this work we show how to perform a deterministic entangling gate between photons by interphasing a system of cold neutral atoms in an optical lattice with a photonic channel; we show that this atomic system can perform at the same time the tasks of storing and processing quantum information. The atomic ensemble is assumed to be in a Mott insulating phase such that the lattice is filled with approximately one atom per site [3]. The photonic input state is mapped to a collective atomic state following lightmatter interface schemes [4]. The ability to control atomic interactions in the optical lattice allows us to perform a gate on collective atomic states which are then released back to the photonic channel. This way, our proposal profits from the advantages of two different experimental techniques which have been recently demonstrated.

Our gate transforms the light input state $\left|\Psi^{\text {in }}\right\rangle_{L}=$ $\alpha|0\rangle_{L}+\beta|1\rangle_{L}+\gamma|2\rangle_{L}$ into $\left|\Psi^{\text {out }}\right\rangle_{L}=\alpha|0\rangle_{L}+i \beta|1\rangle_{L}+$ $\gamma|2\rangle_{L}$, where $|n\rangle_{L}$ is the $n$ photon Fock state. Together with one-qubit rotations this operation is sufficient for universal quantum computation [5]. Each incoming photon creates a collective atomic state, within the subspace of excitations coupling to the light state. This state has to be manipulated such that the resulting state belongs to the same subspace. Using controlled collisions between atoms, this task would require a large number of $O\left(N^{2}\right)$ operations, as each atom has to interact with all others. We face here the problem of implementing efficiently a nonlinear operation with collective states, having only local interactions at our disposal. With our scheme we manage to reduce the number of operations to $O\left(N^{1 / 3}\right)$, by reducing the effective dimensionality of the problem. The main idea is to map collective excitations from the three-dimensional Mott insulator to a plane of particles, then to a line, and finally to a single atom, which can be directly manipulated. The plane, the line, and the single atom are created by means of an initialization protocol, which has to be performed once, before quantum gates can be run on the lattice. Remarkably, our proposal does not require addressability of individual atoms and involves only two internal atomic levels. It comprises four kinds of basic operations, which are all within the experimental state of the art. Finally, the scheme is robust against the main sources of errors in a realistic setup.

Atoms are assumed to possess two internal states $|a\rangle$ and $|b\rangle$ and to be initially prepared in $|a\rangle$. As in quantum memory protocols, for example, in [4], the photonic input state is transferred to the atomic ensemble such that photonic Fock states $|n\rangle_{L}$ are mapped to collective atomic states with $n$ excitations $|n\rangle_{A}$. The initial atomic state is therefore given by $\left|\Psi^{\mathrm{in}}\right\rangle_{A}=\alpha|0\rangle_{A}+\beta|1\rangle_{A}+\gamma|2\rangle_{A}$, where $|1\rangle_{A}$ is a superposition of all permutations of $N$ particle product states containing one atom in $|b\rangle,|1\rangle_{A}=$ $\sum_{j=1}^{N} f_{j}|a\rangle_{1} \ldots|b\rangle_{j} \ldots|a\rangle_{N}$ with $\sum_{j}^{N}\left|f_{j}\right|^{2}=1$. An analogous definition holds for $|2\rangle_{A}$.

Atomic states are processed by means of the following four operations. (1) State-dependent transport. Atoms are displaced depending on their internal state using optical lattices with different polarizations [6,7]. (2) Population transfer between atomic states. Coherent coupling of 
the two atomic levels is achieved by driving Rabi oscillations. A $\pi / 2$ pulse creates the coherent superposition $|a\rangle \mapsto(|a\rangle+|b\rangle) / \sqrt{2},|b\rangle \mapsto(-|a\rangle+|b\rangle) / \sqrt{2}$, while a $\pi$ pulse inverts the atomic population. (3) Collisional phase shift. Controlled collisions between particles in different states are induced by spin dependent transport. If two particles occupy the same lattice site a collisional phase $\phi_{\text {col }}=\Delta E t_{\text {int }}$ is accumulated [7], where $\Delta E$ is the on-site interaction. By controlling the interaction time $t_{\text {int }}, \phi_{\text {col }}$ can be tuned. (4) State-dependent phase shift. A statedependent single particle rotation can be obtained, for example, by applying a magnetic field.

By combining these elements, the two qubit CNOT operation can be implemented (see [7] for details). It transfers a target atom from its initial state $|a\rangle_{t}$ to $|b\rangle_{t}$ if the control atom is in $|b\rangle_{c}$. More specifically, consider control and target atoms placed along the $x$ axis at $x_{c}$ and $x_{t}\left(>x_{c}\right)$, respectively. First, a $\pi / 2$ pulse is applied to the target atom $|a\rangle_{t} \rightarrow\left(|a\rangle_{t}+|b\rangle_{t}\right) / \sqrt{2}$. Then the $|b\rangle$ lattice is displaced along $\hat{x}$, such that the control atom collides with the target atom and induces a $\pi$ phase on $|a\rangle_{t}$. Finally, the initial positions of the atoms are restored and a $-\pi / 2$ pulse is applied to the target atom $\left(-|a\rangle_{t}+|b\rangle_{t}\right) / \sqrt{2} \rightarrow|b\rangle_{t}$.

The key idea in our scheme is to move control atoms in $|b\rangle$ through a set of target atoms in $|a\rangle$, thus transferring the atoms along its path to $|b\rangle$. This tool is employed in two related procedures, which lie at the heart of the scheme. (I) Creation of a d-dimensional structure from $a$ $d$-1-dimensional one. Starting from a control atom in $|b\rangle$ and an ensemble of target atoms in $|a\rangle$, a line of atoms in $|b\rangle$ can be produced by running many CNOT operations in series, such that the control qubit in $|b\rangle$ acts successively on several target atoms in a row, which are accordingly transferred to $|b\rangle$ as explained in Fig. 1 [8]. For the purpose of producing a plane $(d=2)$, we proceed analogously with a line of atoms in $|b\rangle$ instead of a single control qubit. (II) Mapping of collective excitations from an atomic ensemble of dimensionality $d$ to a sample of dimensionality

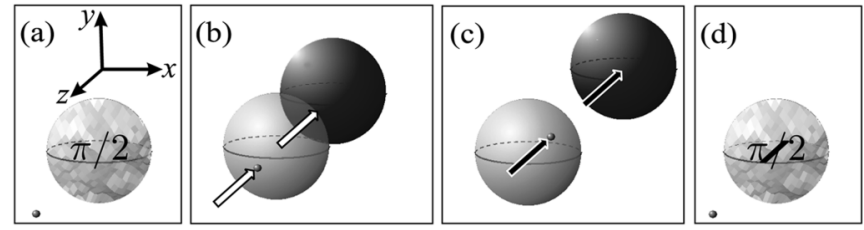

FIG. 1. Creation of a line of atoms in $|b\rangle$. (a) A $\pi / 2$ pulse is applied to the target atoms. (b) $|a\rangle$ and $|b\rangle$ components of the target qubits are separated spatially by a $|b\rangle$ lattice shift along $-\hat{z}$. (c) The $|b\rangle$ lattice is further displaced along $-\hat{z}$, such that the control atom in $|b\rangle$ interacts successively with the $|a\rangle$ part of target atoms along its path, each time leading to a collisional phase $\pi / 2$. Both lattice shifts are reversed leaving all atoms in their original positions. (d) $\mathrm{A}-\pi / 2$ pulse is applied to the target qubits. Atoms which have interacted with the control atom are transferred to $|b\rangle$. $d-1$. A set of control qubits acts upon a set of target qubits. An example is illustrated in Fig. 2, where control atoms in a three-dimensional Mott insulator act on target atoms in a plane. If an atom in the bulk is in state $|b\rangle$, a collision is induced and the target atom hit by the control atom along its path through the plane is transformed to $|b\rangle$. This way atoms in $|b\rangle$ are projected from the bulk to the plane. More precisely, the procedure maps a state with $n$ atoms in $|b\rangle$ to a state with $n$ atoms in $|b\rangle$, except if two atoms in $|b\rangle$ in the bulk are located in a line along $\hat{x}$, leaving the corresponding target atom in $|a\rangle\left(\mathrm{CNOT}^{2}=\right.$ 1 ). In any case an even (odd) number of excitations is mapped to an even (odd) number of excitations in the target object. This method allows us to reduce stepwise the dimensionality of the problem. Finally excitations are mapped from a line to a single site $(d=1)$, and an odd number of excitations in the line transfers the target atom to state $|b\rangle$, while in case of an even number this atom is left in state $|a\rangle$. Thus the parity information is encoded in the state of a single atom.

As mentioned above, the whole scheme can be decomposed into two phases. During the initialization phase, the atoms are divided into four sets, namely, the bulk, a plane, a line, and a dot, which are spatially separated. This setup has to be prepared once and can afterwards be used many times to perform gates. In the second phase the gate protocol itself is performed. We explain first how the setup is prepared and then the processing part. The initialization protocol is summed up in Fig. 3. First a one photon Fock state is mapped to the ensemble [10], resulting in the collective state $|1\rangle_{A}$ with one atom in $|b\rangle$. The atom in $|b\rangle$ can be separated from the surrounding atoms in $|a\rangle$ by applying a global $|b\rangle$ lattice shift. Subsequently the isolated atom is used to create a line of atoms in $|b\rangle$ following procedure (I). Next, this line is separated from the bulk and utilized to produce a plane of atoms in $|b\rangle$ employing the

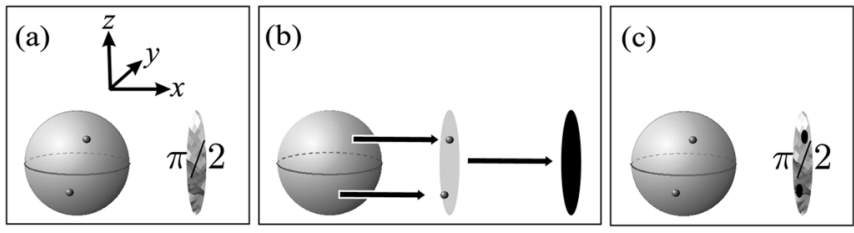

FIG. 2. Mapping of excitations in the bulk to the plane. (a) A $\pi / 2$ pulse is applied to the plane. (b) The $|b\rangle$ lattice is shifted along $\hat{x}$ such that atoms in $|b\rangle$ in the bulk interact with the $|a\rangle$ part of the plane. The time spent after each single-site displacement is chosen such that a phase $\pi / 2$ is accumulated if a collision occurs. Then this lattice movement is reversed. Thus the initial positions of the atoms are restored and each target atom which is located on the path of a control atom in $|b\rangle$ experienced two collisions and picked up a total phase of $\pi$. (c) Finally the plane is subjected to a $-\pi / 2$ pulse, which transfers most of the atoms back to $|a\rangle$. Only atoms, which suffered a collision, are transferred to $|b\rangle$. 


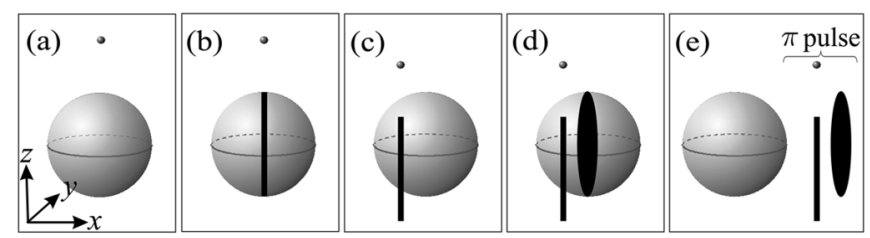

FIG. 3. Initialization of the lattice. (a) A control atom in $|b\rangle$ is placed outside the ensemble. (b) The control qubit interacts successively with a row of target atoms in the ensemble, thus transferring them to $|b\rangle$, as explained in Fig. 1. (c) The resulting line of atoms in $|b\rangle$ is separated from the ensemble along $-\hat{y}$. (d) The line is now used to create a plane of atoms in $|b\rangle$. For this purpose a $\pi / 2$ pulse is applied to the ensemble, collisions are induced by a $|b\rangle$ lattice shift along $\hat{y}$, and a $-\pi / 2$ pulse is applied to the bulk. Since each control atom in the line leads to a line of atoms in $|b\rangle$, which is aligned along $\hat{y}$ we obtain a plane in the $\hat{x} \hat{y}$ plane. (e) The plane is separated from the ensemble by a $|b\rangle$ lattice shift along $\hat{x}$.

same method. Finally the plane is displaced such that the constellation shown in Fig. 3(e) is obtained, and a $\pi$ pulse is applied to the plane, the line, and the dot transferring these atoms to $|a\rangle$. The gate protocol is summarized in Fig. 4. The parity of the number of excitations contained in the bulk is mapped to the dot by means of procedure (II), such that the isolated atom is in state $|b\rangle$ in case of one excitation, while it is in state $|a\rangle$ otherwise. Now a phase shift $\pi / 2$ is applied to the dot if it is in state $|b\rangle$. This way, the atomic state is transformed according to $|0\rangle_{A} \mapsto|0\rangle_{A}$, $|1\rangle_{A} \mapsto i|1\rangle_{A},|2\rangle_{A} \mapsto|2\rangle_{A}$. Then, the previous steps are reversed and the excitations are converted to light, leaving the setup in the original state. Note that none of these steps requires addressability of single sites. Now we consider the truth table corresponding to the protocol. Let us denote by $\left|B_{k}^{n}\right\rangle_{b}$ the initial state of the bulk containing $n=0,1,2$ atoms in $|b\rangle$, located at certain lattice sites according to a configuration $k$ and by $|P\rangle_{p},|L\rangle_{l}$ the state of the plane and the line with all atoms in state $|a\rangle$. Procedure (II) produces the map

$$
\left|B_{k}^{n}\right\rangle_{b}|P\rangle_{p}|L\rangle_{l}|a\rangle_{d} \mapsto\left|B_{k}^{n}\right\rangle_{b}\left|P_{k}^{n^{\prime}}\right\rangle_{p}\left|L_{k}^{n^{\prime \prime}}\right\rangle_{l}\left|a_{n}\right\rangle_{d},
$$

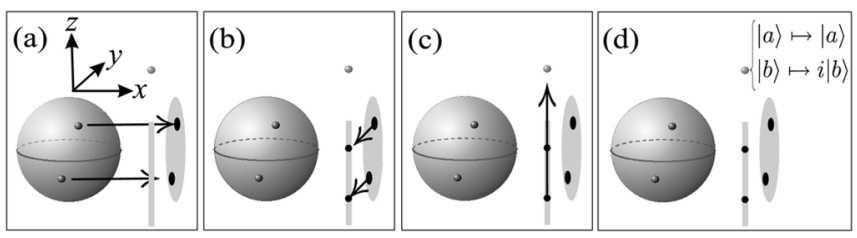

FIG. 4. Quantum gate protocol transforming the input state $\left|\Psi^{\mathrm{in}}\right\rangle_{A}=\alpha|0\rangle_{A}+\beta|1\rangle_{A}+\gamma|2\rangle_{A} \quad$ into $\quad\left|\Psi^{\text {out }}\right\rangle_{A}=\alpha|0\rangle_{A}+$ $i \beta|1\rangle_{A}+\gamma|2\rangle_{A}$. (a)-(c) Excitations in the Mott insulator are successively mapped to structures of lower dimensionality resulting in a single atom being in state $|a\rangle /|b\rangle$ in case of an even/ odd number of excitations in the Mott insulator. (d) A statedependent phase is applied to the isolated particle such that $|1\rangle_{A} \mapsto i|1\rangle_{A}$. Subsequently steps (a)-(c) have to be reversed. where $\left|P_{k}^{n^{\prime}}\right\rangle_{p}$ and $\left|L_{k}^{n^{\prime \prime}}\right\rangle_{l}$ refer to the states of the plane and line after the excitations have been mapped and $\left|a_{n}\right\rangle_{d}$ describes the state of the dot with $a_{0}=a_{2}=a$ and $a_{1}=$ $b$. Thus, the whole protocol results in

$$
\left|B_{k}^{n}\right\rangle_{b}|P\rangle_{p}|L\rangle_{l}|a\rangle_{d} \mapsto i^{n \bmod 2}\left|B_{k}^{n}\right\rangle_{b}|P\rangle_{p}|L\rangle_{l}|a\rangle_{d}
$$

Note that collective excitations are not localized, but a superposition of states with atoms in $|b\rangle$ at different sites. Moreover, we have a superposition of different positions of the plane, the line, and the dot, as the excitation created at the beginning of the initialization is also delocalized. For any term in the superposition, the final state differs only in a phase from the initial state. By adding the terms in Eq. (1) with respect to the positions of the excitations, $k$, and the positions of the plane, the line, and the dot we obtain the desired gate transformation. We remark that the protocol does not rely on a coherent superposition of the different positions of the dot, the line, and the plane. It can also be run using a mixed state.

In the following we analyze the main sources of errors in our scheme [11]. It has been carefully designed in order to minimize decoherence, first of all, by avoiding the presence of cat states in the internal atomic states, which would give rise to errors if few particles are lost. Apart from that, run times are very short such that decoherence has not much time to act. In particular, the run time is essentially given by the time needed to run the collisional steps, since population transfers and separations can be done much faster. Each collisonal step has to be run along a whole ensemble length and requires therefore a time $t_{\text {int }} N^{1 / 3}$, where $t_{\text {int }}$ is the time spent in a single collision. Remarkably, the three-dimensional problem scales like a one-dimensional one in time, since the task of scanning $N$ particles in three dimensions is accomplished by a onedimensional projection scheme.

We address now transitions from $|a\rangle$ to $|b\rangle$ or to another trapped state affected by $|b\rangle$ lattice shifts and give an example how a judicious choice of atomic levels allows us to sidestep this source of errors, while still being able to perform the operations necessary for the quantum gate. By employing alkali atoms with nuclear spin $1 / 2$, for instance, the qubit can be encoded in hyperfine states of the $S_{1 / 2}$ shell by identifying $|a\rangle \equiv\left|F=1, m_{F}=-1\right\rangle$ and $|b\rangle \equiv$ $\left|F=1, m_{F}=1\right\rangle$. By choosing appropriately the detuning of two off-resonant standing waves with different polarizations [7], state-dependent transport can be implemented by trapping $\left|F=1, m_{F}=-1\right\rangle$ and $\left|F=1, m_{F}=1\right\rangle$ by $\sigma_{-}$and $\sigma_{+}$light, respectively. Transitions $|a\rangle \mapsto|b\rangle$ cannot be induced by means of the off-resonant laser fields, since $|a\rangle$ corresponds to the nuclear magnetic quantum number $m_{I}=-1 / 2$, while $|b\rangle$ corresponds to $m_{I}=1 / 2$. $\pi / 2$ or $\pi$ pulses can be applied by means of resonant twophoton Raman or microwave transitions. Finally, the standing waves do induce transitions to the other trapped states 
$\left|F=1, m_{F}=0\right\rangle$ and $\left|F=0, m_{F}=0\right\rangle$. However, the optical potential experienced by these levels is given by the equally weighted sum of contributions from both polarizations. While shifting one lattice with respect to the other, the optical potential vanishes at some point, and these two levels are emptied, which ensures that they do not affect the protocol.

Among the remaining noise mechanisms, the most important ones are imperfect population transfer and dephasing of quantum states [11] between two $\pi / 2$ pulses. The corresponding probability of error is proportional to the number of target atoms in the mapping steps $N^{2 / 3}$. This failure probability can be reduced by using an elongated ensemble having a spatial extend $L$ along the direction of the first shift in the gate protocol and a length $l<L$ along the other directions. In this case the probability of obtaining a wrong result is proportional to $l^{2}$.

The probability of error due to the remaining noise mechanism scales at worst like $N^{1 / 3}$, i.e., proportional to the run time. First we consider imperfections in the $\pi$ pulse, performed at the end of the initialization. Since an imperfect population transfer leaves atoms in a superposition state, the $|b\rangle$ lattice should be emptied as an additional step after the $\pi$ pulse. Another source of errors are occupation number defects. We only have to deal with empty lattice sites, since double occupied sites can be avoided by choosing low filling factors. Holes in the plane and the line lead to a wrong result, if they are located at specific sites which interact with an atom in $|b\rangle$ in the course of the protocol. The failure probability due to defects which are initially present in the Mott insulator are given by the probability for a single site to be unoccupied, and it does not depend on the size of the system. Holes can also be created as a consequence of atomic transitions into untrapped states. This dynamical particle loss induces an error which scales like the duration of the gate, $N^{1 / 3}$. Another limiting factor are imperfect collisions. The phase acquired in each shift during the collisional steps may differ from $\phi_{\text {col }}=\pi$. As in the case of unoccupied lattice sites, the probability of obtaining a wrong result is given by the probability on the single-site level. The fidelity of the scheme is also decreased by undesired collisional phases. The corresponding failure probability is proportional to $N^{1 / 3}$, since these phases are accumulated in onedimensional operations each covering one ensemble length. Finally, kinetic phases acquired by the atoms during lattice shifts do not play a role in the proposed scheme. Employing the common technique for state-dependent transport, the nodes of two optical potentials forming standing waves are moved in opposite directions $V_{ \pm}(x)=$ $\cos ^{2}(k x \pm \phi)$ for some wave vector $k$, spatial variable $x$, and angle $\phi$. Lattice shifts affect therefore both atomic species in the same way and lead only to global phases of the resulting states.

We thank Eugene Polzik for discussions and acknowledge support from the Elite Network of Bavaria (ENB) project QCCC, the EU projects SCALA and COVAQUIAL, the DFG-Forschungsgruppe No. 635, and Ministerio de Educacion y Ciencia No. EX-2006-0295.

[1] W. Dür, H.-J. Briegel, J. I. Cirac, and P. Zoller, Phys. Rev. A 59, 169 (1999); L.-M. Duan, J. I. Cirac, M. Lukin, and P. Zoller, Nature (London) 414, 413 (2001).

[2] E. Knill, R. Laflamme, and G. J. Milburn, Nature (London) 409, 46 (2001).

[3] D. Jaksch, C. Bruder, J. I. Cirac, C. W. Gardiner, and P. Zoller, Phys. Rev. Lett. 81, 3108 (1998); M. Greiner, O. Mandel, T. Esslinger, T. W. Hänsch, and I. Bloch, Nature (London) 415, 39 (2002).

[4] B. Julsgaard, J. Sherson, J. I. Cirac, J. Fiurasek, and E. Polzik, Nature (London) 432, 482 (2004); C. W. Chou, H. de Riedmatten, D. Felinto, S. V. Polyakov, S. J. van Enk, and H. J. Kimble, Nature (London) 438, 828 (2005); T. Chanelière, D. N. Matsukevich, S. D. Jenkins, S.-Y. Lan, T. A. B. Kennedy, and A. Kuzmich, Nature (London) 438, 833 (2005); M. D. Eisaman, A. Andre, F. Massou, M. Fleischhauer, A.S. Zibrov, and M.D. Lukin, Nature (London) 438, 837 (2005).

[5] A. Barenco, C. H. Bennett, R. Cleve, D. P. DiVincenzo, N. Margolus, P. Shor, T. Sleator, J. A. Smolin, and H. Weinfurter, Phys. Rev. A, 52, 3457 (1995).

[6] G. K. Brennen, C. M. Caves, P.S. Jessen, and I. H. Deutsch, Phys. Rev. Lett. 82, 1060 (1999).

[7] D. Jaksch, H.-J. Briegel, J. I. Cirac, C. W. Gardiner, and P. Zoller, Phys. Rev. Lett. 82, 1975 (1999); O. Mandel, M. Greiner, A. Widera, T. Rom, T. W. Hänsch, and I. Bloch, Nature (London) 425, 937 (2003).

[8] The separation step shown in Fig. 1(b) can be performed either by displacing the lattices first by half a lattice spacing along $\hat{x}$ (or $\hat{y}$ ) and then by a distance exceeding the length of the ensemble along $-\hat{z}$, or by moving the lattice fast along $-\hat{z}$, which can be done such the atoms start and end up in their motional ground state [9].

[9] H.-J. Briegel, T. Calarco, D. Jaksch, J. I. Cirac, and P. Zoller, J. Mod. Opt. 47, 415 (2000); U. Dorner, T. Calarco, P. Zoller, A. Browaeys, and P. Grangier, J. Opt. B 7, S341 (2005).

[10] This can, for example, be done using heralded single photons from an EPR source or a weak coherent field together with a postselecting photon detection.

[11] A quantitative analysis will be given elsewhere. 